\title{
SUPPLEMENTARY MATERIAL: A KNOCKOFF FILTER FOR HIGH-DIMENSIONAL SELECTIVE INFERENCE
}

\author{
By Rina Foygel BARber AND EMMANUEL J. CANDÈS
}

1. Additional proofs. In this section, we first prove Lemma 1 (presented in Section 3 of the main paper) and some additional supporting lemmas, and then present the proof of our general error control result, Theorem 4 (presented in Appendix A.1 of the main paper).

1.1. Key lemmas. The proof of Theorem 4 relies on two key lemmas: Lemma 1 earlier which treats non-i.i.d. Bernoulli sequences, and the following result regarding the statistics $\mathbf{W}$ arising in the knockoff procedure.

LEMMA 1. With the assumptions from Theorem 4, let $\mathcal{V}$ be the $\sigma$-algebra generated by the random variables $\left\{(\mathbf{X}+\widetilde{\mathbf{X}})^{\top} \mathbf{y},\left|(\mathbf{X}-\widetilde{\mathbf{X}})^{\top} \mathbf{y}\right|\right\}$, and define the vector $\mathbf{S}=\left(S_{1}, \ldots, S_{p}\right)$ of signs as

$$
S_{j}=\operatorname{sign}\left(\left(\mathbf{X}_{j}-\widetilde{\mathbf{X}}_{j}\right)^{\top} \mathbf{y}\right) \cdot \operatorname{sign}\left(W_{j}\right) .
$$

Then the following holds:

- $|\mathbf{W}|$ and $\mathbf{S}$ belong to $\mathcal{V}$.

- The signs of $W_{j}$ are mutually independent after conditioning on $\mathcal{V}$.

- For each $j=1, \ldots, p$, if $S_{j} \neq \operatorname{sign}\left(\left(\mathbf{X}_{j}-\widetilde{\mathbf{X}}_{j}\right)^{\top} \boldsymbol{\mu}\right)$, then

$$
\mathbb{P}\left\{\operatorname{sign}\left(W_{j}\right)=-1 \mid \mathcal{V}\right\} \geq 1 / 2 .
$$

Before presenting the formal proof, we give an intuitive explanation for why the probabilities for $\operatorname{sign}\left(W_{j}\right)$ work in our favor; that is, why we are able to obtain $\mathbb{P}\left\{\operatorname{sign}\left(W_{j}\right)=-1 \mid \mathcal{V}\right\} \geq 1 / 2$, which ensures that we are more likely to overrather than under-estimate the number of false positives. This is similar to the discussion for the low-dimensional setting as in (15). In this case, for the highdimensional setting, we see that if $S_{j} \neq \operatorname{sign}\left(\left(\mathbf{X}_{j}-\widetilde{\mathbf{X}}_{j}\right)^{\top} \boldsymbol{\mu}\right)$, then:

- If $\left(\mathbf{X}_{j}-\widetilde{\mathbf{X}}_{j}\right)^{\top} \boldsymbol{\mu} \geq 0$, then $\operatorname{sign}\left(W_{j}\right)=-1$ whenever $\left(\mathbf{X}_{j}-\widetilde{\mathbf{X}}_{j}\right)^{\top} \mathbf{y}>0$;

- If $\left(\mathbf{X}_{j}-\widetilde{\mathbf{X}}_{j}\right)^{\top} \boldsymbol{\mu} \leq 0$, then $\operatorname{sign}\left(W_{j}\right)=-1$ whenever $\left(\mathbf{X}_{j}-\widetilde{\mathbf{X}}_{j}\right)^{\top} \mathbf{y}<0$.

Of course, $\left(\mathbf{X}_{j}-\widetilde{\mathbf{X}}_{j}\right)^{\top} \mathbf{y}$ is a normal random variable whose mean is given by $\left(\mathbf{X}_{j}-\widetilde{\mathbf{X}}_{j}\right)^{\top} \boldsymbol{\mu}$. If this mean is nonnegative, then the random variable $\left(\mathbf{X}_{j}-\widetilde{\mathbf{X}}_{j}\right)^{\top} \mathbf{y}$ 
is more likely to be positive than negative; if this mean is nonpositive, then the opposite is true. Either way, we see that $\operatorname{sign}\left(W_{j}\right)=-1$ has probability at least $1 / 2$.

Proof. First, by the sufficiency property, $\mathbf{W}$ is a function of $\left[\begin{array}{ll}\mathbf{X} & \widetilde{\mathbf{X}}\end{array}\right]^{\top}\left[\begin{array}{ll}\mathbf{X} & \widetilde{\mathbf{X}}\end{array}\right]$ and $\left[\begin{array}{ll}\mathbf{X} & \widetilde{\mathbf{X}}\end{array}\right]^{\top} \mathbf{y}$ or, equivalently, is of the form

$$
\mathbf{W}=\mathbf{w}\left(\left[\begin{array}{ll}
\mathbf{X} & \widetilde{\mathbf{X}}
\end{array}\right]^{\top}[\mathbf{X} \quad \tilde{\mathbf{X}}],(\mathbf{X}+\widetilde{\mathbf{X}})^{\top} \mathbf{y},\left|(\mathbf{X}-\widetilde{\mathbf{X}})^{\top} \mathbf{y}\right|, \operatorname{sign}\left((\mathbf{X}-\widetilde{\mathbf{X}})^{\top} \mathbf{y}\right)\right)
$$

for some function $\mathbf{w}$. Second, the antisymmetry property implies that flipping the signs of any subset of the last argument of the function $\mathbf{w}$ flips the signs of the corresponding outputs. (Of course, for any $j$ such that $\left(\mathbf{X}_{j}-\widetilde{\mathbf{X}}_{j}\right)^{\top} \mathbf{y}=0$, the antisymmetry property implies that $W_{j}=0$; we ignore these features in our proof from this point on as they can never be selected by our method.) This property makes clear that $\mathcal{V}$ determines $\mathbf{W}$ up to a sign change, so that $|\mathbf{W}| \in \mathcal{V}$. Furthermore, define a vector of signs $\mathbf{S}$ such that

$$
|\mathbf{W}|=\mathbf{w}\left(\left[\begin{array}{ll}
\mathbf{X} & \widetilde{\mathbf{X}}
\end{array}\right]^{\top}\left[\begin{array}{ll}
\mathbf{X} & \widetilde{\mathbf{X}}
\end{array}\right],(\mathbf{X}+\widetilde{\mathbf{X}})^{\top} \mathbf{y},\left|(\mathbf{X}-\widetilde{\mathbf{X}})^{\top} \mathbf{y}\right|, \mathbf{S}\right) .
$$

Clearly, $\mathbf{S} \in \mathcal{V}$ and, by the antisymmetry property, we also see that

$$
\mathbf{w}\left(\left[\begin{array}{ll}
\mathbf{X} & \widetilde{\mathbf{X}}
\end{array}\right]^{\top}\left[\begin{array}{ll}
\mathbf{X} & \widetilde{\mathbf{X}}
\end{array}\right],(\mathbf{X}+\widetilde{\mathbf{X}})^{\top} \mathbf{y},\left|(\mathbf{X}-\widetilde{\mathbf{X}})^{\top} \mathbf{y}\right|, \mathbf{S} \cdot \operatorname{sign}(\mathbf{W})\right)=\mathbf{W} .
$$

This equality means that $\mathbf{S} \cdot \operatorname{sign}(\mathbf{W})$ must be equal to $\operatorname{sign}\left((\mathbf{X}-\widetilde{\mathbf{X}})^{\top} \mathbf{y}\right)$ or, expressed differently, $\mathbf{S}=\operatorname{sign}(\mathbf{W}) \cdot \operatorname{sign}\left((\mathbf{X}-\widetilde{\mathbf{X}})^{\top} \mathbf{y}\right)$ thereby coinciding with the definition given in the statement of the lemma. This establishes the first claim.

Next, an elementary calculation shows that

$$
\left[\begin{array}{l}
(\mathbf{X}+\widetilde{\mathbf{X}})^{\top} \mathbf{y} \\
(\mathbf{X}-\widetilde{\mathbf{X}})^{\top} \mathbf{y}
\end{array}\right] \sim \mathcal{N}\left(\left[\begin{array}{l}
(\mathbf{X}+\widetilde{\mathbf{X}})^{\top} \boldsymbol{\mu} \\
(\mathbf{X}-\widetilde{\mathbf{X}})^{\top} \boldsymbol{\mu}
\end{array}\right],\left[\begin{array}{cc}
(\mathbf{X}+\widetilde{\mathbf{X}})^{\top} \boldsymbol{\Theta}(\mathbf{X}+\widetilde{\mathbf{X}}) & 0 \\
0 & \operatorname{diag}\{\mathbf{d}\}
\end{array}\right]\right)
$$

where $\mathbf{d}=\left(d_{1}, \ldots, d_{p}\right) \in \mathbb{R}^{p}$ is some nonnegative vector. The form of the covariance term arises from the fact that $(\mathbf{X}-\widetilde{\mathbf{X}})^{\top} \boldsymbol{\Theta}(\mathbf{X}+\widetilde{\mathbf{X}})=0$ and $(\mathbf{X}-$ $\widetilde{\mathbf{X}})^{\top} \boldsymbol{\Theta}(\mathbf{X}-\widetilde{\mathbf{X}})$ is diagonal, according to the pairwise exchangeability assumption on $\left[\begin{array}{ll}\mathbf{X} & \widetilde{\mathbf{X}}\end{array}\right]^{\top} \boldsymbol{\Theta}\left[\begin{array}{ll}\mathbf{X} & \widetilde{\mathbf{X}}\end{array}\right]$. Therefore, the terms $\left(\mathbf{X}_{j}-\widetilde{\mathbf{X}}_{j}\right)^{\top} \mathbf{y}$ are mutually independent, and are independent from $(\mathbf{X}+\widetilde{\mathbf{X}})^{\top} \mathbf{y}$. Also, since $\mathbf{S} \in \mathcal{V}$ the terms $\operatorname{sign}\left(W_{j}\right)=S_{j} \cdot \operatorname{sign}\left(\left(\mathbf{X}_{j}-\widetilde{\mathbf{X}}_{j}\right)^{\top} \mathbf{y}\right)$ are mutually independent after conditioning on $\mathcal{V}$, proving our second claim. 
Finally, observe that if $Z \sim \mathcal{N}\left(\theta, \sigma^{2}\right)$, then it follows from a simple calculation that

$$
\frac{\mathbb{P}\{\operatorname{sign}(Z)=-1|| Z \mid\}}{\mathbb{P}\{\operatorname{sign}(Z)=+1|| Z \mid\}}=\exp \left(-\frac{2 \theta|z|}{\sigma^{2}}\right)
$$

Now since $\mathbb{P}\left\{\operatorname{sign}\left(W_{j}\right)= \pm 1 \mid \mathcal{V}\right\}=\mathbb{P}\left\{S_{j} \cdot \operatorname{sign}\left(\left(\mathbf{X}_{j}-\widetilde{\mathbf{X}}_{j}\right)^{\top} \mathbf{y}\right)= \pm 1 \mid \mathcal{V}\right\}$, applying the above formula gives

$$
\frac{\mathbb{P}\left\{\operatorname{sign}\left(W_{j}\right)=-1 \mid \mathcal{V}\right\}}{\mathbb{P}\left\{\operatorname{sign}\left(W_{j}\right)=+1 \mid \mathcal{V}\right\}}=\exp \left(-\frac{\left.2 S_{j} \cdot\left(\mathbf{X}_{j}-\widetilde{\mathbf{X}}\right)^{\top} \boldsymbol{\mu} \cdot \mid\left(\mathbf{X}_{j}-\widetilde{\mathbf{X}}_{j}\right)^{\top} \mathbf{y}\right) \mid}{d_{j}}\right)
$$

Clearly, if $S_{j} \neq \operatorname{sign}\left(\left(\mathbf{X}_{j}-\widetilde{\mathbf{X}}_{j}\right)^{\top} \boldsymbol{\mu}\right)$, then the argument in the exponential in the right-hand side of this last inequality is positive and thus, $\mathbb{P}\left\{\operatorname{sign}\left(W_{j}\right)=-1 \mid \mathcal{V}\right\} \geq$ $1 / 2$.

The original knockoff paper [1] worked in the simpler setting where the signs of $W_{j}$ for the nulls are i.i.d. unbiased. If $\mathcal{H}_{0}$ is the set of nulls and $T>0$ the adaptive threshold of the knockoff filter, [1] established that the i.i.d. property for the signs gives

$$
\mathbb{E}\left[\mathbb{E}\left[\frac{\left|\left\{j \in \mathcal{H}_{0}: W_{j} \geq T\right\}\right|}{1+\left|\left\{j \in \mathcal{H}_{0}: W_{j} \leq-T\right\}\right|}|| \mathbf{W} \mid\right]\right] \leq 1 .
$$

Here, we use Lemma 1 to develop a similar bound when the signs are no longer i.i.d. unbiased but are, instead, independent with at most a $50 \%$ chance of being positive conditionally on $\mathcal{V}$.

Corollary 1. Suppose $\mathcal{M} \subset\{1, \ldots, p\}$ is a random set belonging to $\mathcal{V}$ and chosen such that for each $j \in \mathcal{M},\left|W_{j}\right|>0$ and $\mathbb{P}\left\{\operatorname{sign}\left(W_{j}\right)=-1 \mid \mathcal{V}\right\} \geq \rho$. Letting $T>0$ be the adaptive threshold of either the knockoff or knockoff+ filter, then

$$
\mathbb{E}\left[\frac{\left|\left\{j \in \mathcal{M}: W_{j} \geq T\right\}\right|}{1+\left|\left\{j \in \mathcal{M}: W_{j} \leq-T\right\}\right|} \mid \mathcal{V}\right] \leq \rho^{-1}-1
$$

PROOF. The proof is straightforward and only consists in rewriting the left-hand side of (2) in such a way that we recognize the formulation from Lemma 1. To begin with, we can treat the ordering of $|\mathbf{W}|$ as fixed since Lemma 1 gives $|\mathbf{W}| \in \mathcal{V}$. Now assume without loss of generality that $\mathcal{M}=\{1, \ldots, m\}$ and reorder the indices of the $W_{j}$ 's in $\mathcal{M}$ so that $\left|W_{(1)}\right| \geq\left|W_{(2)}\right| \geq \cdots \geq\left|W_{(m)}\right|$. Set $B_{j}=\mathbb{1}_{W_{(j)}<0}$. Next we will condition also on $\operatorname{sign}\left(W_{j}\right)$ for all $j \notin \mathcal{M}$. Let $\widetilde{\mathcal{V}}$ be the larger $\sigma$-algebra generated by $\mathcal{V}$ and $\left\{\operatorname{sign}\left(W_{j}\right): j \notin \mathcal{M}\right\}$. By definition of $\mathcal{M}$, when we condition on $\mathcal{V}$, we have $B_{j} \stackrel{\Perp}{\sim} \operatorname{Bernoulli}\left(\rho_{j}\right)$ with $\rho_{j} \geq \rho$ for all $j \in \mathcal{M}$. By Lemma 1 , the 
same is still true even after conditioning on $\left\{\operatorname{sign}\left(W_{j}\right): j \notin \mathcal{M}\right\}$ also, since the signs of $\mathbf{W}$ are mutually independent conditional on $\mathcal{V}$. To summarize, we have

$$
\left(B_{j}\right)_{j \in \mathcal{M}} \mid \widetilde{\mathcal{V}} \stackrel{\Perp}{\sim} \operatorname{Bernoulli}\left(\rho_{j}\right),
$$

with $\rho_{j} \geq \rho$ for all $j \in \mathcal{M}$.

Next, we can write

$\frac{\#\left\{j \in \mathcal{M}: W_{j} \geq T\right\}}{1+\#\left\{j \in \mathcal{M}: W_{j} \leq-T\right\}}=\frac{\left(1-B_{1}\right)+\cdots+\left(1-B_{J}\right)}{1+B_{1}+\cdots+B_{J}}=\frac{1+J}{1+B_{1}+\cdots+B_{J}}-1$,

where $J$ is the index such that $\left|W_{(1)}\right| \geq \cdots \geq\left|W_{(J)}\right| \geq T>\left|W_{(J+1)}\right| \geq \cdots \geq$ $\left|W_{(m)}\right|$. After conditioning on $\widetilde{\mathcal{V}}$, so that we can treat $|\mathbf{W}|$ and $\left\{\operatorname{sign}\left(W_{j}\right): j \notin\right.$ $\mathcal{M}\}$ as fixed, we observe that the index $J$ can be expressed as a stopping time, in reverse time, with respect to the filtration $\left\{\mathcal{F}_{j}\right\}$ given by

$$
\mathcal{F}_{j}=\left\{B_{1}+\cdots+B_{j}, B_{j+1}, \ldots, B_{m}\right\} .
$$

The conclusion now follows from Lemma 1.

1.2. Proof of Theorem 4. First, consider control of $\mathrm{mFDR}_{\mathrm{dir}}$ under the knockoff. Since $j \in \widehat{S}$ if and only if $W_{j} \geq T$ where $T$ is our adaptive threshold, the modified directional FDR is given by

$$
\begin{aligned}
\operatorname{mFDR}_{\mathrm{dir}} & =\mathbb{E}\left[\frac{\left|\left\{j: W_{j} \geq T, \widehat{\operatorname{sign}}_{j} \neq \operatorname{sign}\left(\left(\mathbf{X}_{j}-\widetilde{\mathbf{X}}_{j}\right)^{\top} \boldsymbol{\mu}\right)\right\}\right|}{\left|\left\{j: W_{j} \geq T\right\}\right|+q^{-1}}\right] \\
& =\mathbb{E}\left[\frac{\left|\left\{j: W_{j} \geq T, \widehat{\operatorname{sign}}_{j} \neq \operatorname{sign}\left(\left(\mathbf{X}_{j}-\widetilde{\mathbf{X}}_{j}\right)^{\top} \boldsymbol{\mu}\right)\right\}\right|}{1+\left|\left\{j: W_{j} \leq-T\right\}\right|} \cdot \frac{1+\left|\left\{j: W_{j} \leq-T\right\}\right|}{\left|\left\{j: W_{j} \geq T\right\}\right|+q^{-1}}\right] \\
& \leq q \cdot \mathbb{E}\left[\frac{\left|\left\{j: W_{j} \geq T, \widehat{\operatorname{sign}}_{j} \neq \operatorname{sign}\left(\left(\mathbf{X}_{j}-\widetilde{\mathbf{X}}_{j}\right)^{\top} \boldsymbol{\mu}\right)\right\}\right|}{1+\left|\left\{j: W_{j} \leq-T\right\}\right|}\right]
\end{aligned}
$$

since $\left|\left\{j: W_{j} \leq-T\right\}\right| \leq q \cdot\left|\left\{j: W_{j} \geq T\right\}\right|$ by definition of $T$. Now rewrite the set of sign errors as

$$
\begin{aligned}
& \left\{j: W_{j} \geq T, \widehat{\operatorname{sign}}{ }_{j} \neq \operatorname{sign}\left(\left(\mathbf{X}_{j}-\widetilde{\mathbf{X}}_{j}\right)^{\top} \boldsymbol{\mu}\right)\right\}=\left\{j: W_{j} \geq T, \operatorname{sign}\left(\left(\mathbf{X}_{j}-\widetilde{\mathbf{X}}_{j}\right)^{\top} \mathbf{y}\right) \neq \operatorname{sign}\left(\left(\mathbf{X}_{j}-\widetilde{\mathbf{X}}_{j}\right)^{\top} \boldsymbol{\mu}\right)\right\} \\
& =\left\{j: W_{j} \geq T, S_{j} \neq \operatorname{sign}\left(\left(\mathbf{X}_{j}-\widetilde{\mathbf{X}}_{j}\right)^{\top} \boldsymbol{\mu}\right)\right\}
\end{aligned}
$$

where the first equality follows from the definition of $\widehat{\operatorname{sign}}_{j}$ in (15), and the second from the definition $S_{j}=\operatorname{sign}\left(\left(\mathbf{X}_{j}-\widetilde{\mathbf{X}}_{j}\right)^{\top} \mathbf{y}\right) \cdot \operatorname{sign}\left(W_{j}\right)$ as in Lemma 1 (since 
$\operatorname{sign}\left(W_{j}\right)=+1$ for all $j$ in this set $)$. Define $\widehat{\mathcal{H}_{0}}=\left\{j: S_{j} \neq \operatorname{sign}\left(\left(\mathbf{X}_{j}-\widetilde{\mathbf{X}}_{j}\right)^{\top} \boldsymbol{\mu}\right)\right\}$ and let $\mathcal{V}$ be as in Lemma 1 . By the tower law we obtain

$$
\begin{aligned}
\operatorname{mFDR}_{\mathrm{dir}} & \leq q \cdot \mathbb{E}\left[\mathbb{E}\left[\frac{\left|\left\{j: W_{j} \geq T, S_{j} \neq \operatorname{sign}\left(\left(\mathbf{X}_{j}-\widetilde{\mathbf{X}}_{j}\right)^{\top} \boldsymbol{\mu}\right)\right\}\right|}{1+\left|\left\{j: W_{j} \leq-T\right\}\right|} \mid \mathcal{V}\right]\right] \\
& =q \cdot \mathbb{E}\left[\mathbb{E}\left[\frac{\left|\left\{j \in \widehat{\mathcal{H}_{0}}: W_{j} \geq T\right\}\right|}{1+\left|\left\{j: W_{j} \leq-T\right\}\right|}|\mathcal{V}|\right]\right. \\
& \leq q \cdot \mathbb{E}\left[\mathbb{E}\left[\frac{\left|\left\{j \in \widehat{\mathcal{H}_{0}}: W_{j} \geq T\right\}\right|}{1+\left|\left\{j \in \widehat{\mathcal{H}_{0}}: W_{j} \leq-T\right\}\right|} \mid \mathcal{V}\right]\right] .
\end{aligned}
$$

Next, note that $\widehat{\mathcal{H}_{0}} \in \mathcal{V}$ since $\mathbf{S} \in \mathcal{V}$ by Lemma 1 , and so inside of the conditional expectation, we can treat the set $\widehat{\mathcal{H}_{0}}$ as fixed. Lemma 1 proves that conditional on $\mathcal{V}$, the signs of $\mathbf{W}$ are independent, with $\mathbb{P}\left\{\operatorname{sign}\left(W_{j}\right)=-1 \mid \mathcal{V}\right\} \geq 1 / 2$ for all $j \in \widehat{\mathcal{H}_{0}}$. Therefore, applying Corollary 1 with $\rho=1 / 2$ gives

$$
\mathbb{E}\left[\frac{\left|\left\{j \in \widehat{\mathcal{H}_{0}}: W_{j} \geq T\right\}\right|}{1+\left|\left\{j \in \widehat{\mathcal{H}_{0}}: W_{j} \leq-T\right\}\right|} \mid \mathcal{V}\right] \leq 1,
$$

which concludes the argument.

The proof for FDR $\mathrm{dir}_{\text {, }}$ when using knockoff+ instead of knockoff, follows similarly: we have

$$
\begin{aligned}
\mathrm{FDR}_{\mathrm{dir}} & =\mathbb{E}\left[\frac{\left|\left\{j: W_{j} \geq T_{+}, \widehat{\operatorname{sign}}_{j} \neq \operatorname{sign}\left(\left(\mathbf{X}_{j}-\widetilde{\mathbf{X}}_{j}\right)^{\top} \boldsymbol{\mu}\right)\right\}\right|}{1+\left|\left\{j: W_{j} \leq-T_{+}\right\}\right|} \cdot \frac{1+\left|\left\{j: W_{j} \leq-T_{+}\right\}\right|}{1 \vee\left|\left\{j: W_{j} \geq T_{+}\right\}\right|}\right] \\
& \leq q \cdot \mathbb{E}\left[\frac{\left|\left\{j: W_{j} \geq T_{+}, \widehat{\operatorname{sign}}_{j} \neq \operatorname{sign}\left(\left(\mathbf{X}_{j}-\widetilde{\mathbf{X}}_{j}\right)^{\top} \boldsymbol{\mu}\right)\right\}\right|}{1+\left|\left\{j: W_{j} \leq-T_{+}\right\}\right|}\right],
\end{aligned}
$$

where the last step uses the (slightly more conservative) definition of the threshold $T_{+}$for the knockoff+ procedure. From then on, the argument is the same as before.

1.3. Proof of Lemma 1. We first give a slight generalization of Lemma 4 in [1]:

LEMMA 2. Suppose that $B_{1}, \ldots, B_{n} \stackrel{\mathrm{iid}}{\sim} \operatorname{Bernoulli}(\rho)$. Let $J$ be a stopping time in reverse time with respect to the filtration $\left\{\mathcal{F}_{j}\right\}$, where $\mathcal{F}_{j} \ni B_{1}+\cdots+B_{j}, B_{j+1}, \ldots, B_{n}$, and where the variables $B_{1}, \ldots, B_{j}$ are exchangeable with respect to $\mathcal{F}_{j}$. Then

$$
\mathbb{E}\left[\frac{1+J}{1+B_{1}+\cdots+B_{J}}\right] \leq \rho^{-1}
$$


Proof of Lemma 2. Define the sum

$$
S_{j}=B_{1}+\cdots+B_{j} \in \mathcal{F}_{j}
$$

and define the process

$$
M_{j}=\frac{1+j}{1+B_{1}+\cdots+B_{j}}=\frac{1+j}{1+S_{j}} \in \mathcal{F}_{j} .
$$

In [1] it is shown that $\mathbb{E}\left[M_{n}\right] \leq \rho^{-1}$, therefore, by the optional stopping time theorem it suffices to show that $\left\{M_{j}\right\}$ is a supermartingale with respect to $\left\{\mathcal{F}_{j}\right\}$. First, since $\left\{B_{1}, \ldots, B_{j+1}\right\}$ are exchangeable with respect to $\mathcal{F}_{j+1}$, we have

$$
\mathbb{P}\left\{B_{j+1}=1 \mid \mathcal{F}_{j+1}\right\}=\frac{S_{j+1}}{1+j} .
$$

Therefore, if $S_{j+1}>0$,

$$
\begin{aligned}
\mathbb{E}\left[M_{j} \mid \mathcal{F}_{j+1}\right] & =\frac{1+j}{1+S_{j+1}} \cdot \mathbb{P}\left\{B_{j+1}=0 \mid \mathcal{F}_{j+1}, S_{j+1}\right\}+\frac{1+j}{1+S_{j+1}-1} \cdot \mathbb{P}\left\{B_{j+1}=1 \mid \mathcal{F}_{j+1}, S_{j+1}\right\} \\
& =\frac{1+j}{1+S_{j+1}} \cdot \frac{1+j-S_{j+1}}{1+j}+\frac{1+j}{1+S_{j+1}-1} \cdot \frac{S_{j+1}}{1+j} \\
& =\frac{1+j-S_{j+1}}{1+S_{j+1}}+1=\frac{1+(j+1)}{1+S_{j+1}}=M_{j+1} .
\end{aligned}
$$

If instead $S_{j+1}=0$, then trivially $S_{j}=0$ also, and so $M_{j}=1+j<2+j=M_{j+1}$. This proves that $\left\{M_{j}\right\}$ is a supermartingale with respect to $\left\{\mathcal{F}_{j}\right\}$, as desired.

COROLlary 2. Suppose that $\mathcal{A} \subseteq[n]$ is fixed, while $B_{1}, \ldots, B_{n} \stackrel{\text { iid }}{\sim} \operatorname{Bernoulli}(\rho)$. Let $J$ be a stopping time in reverse time with respect to the filtration $\left\{\mathcal{F}_{j}\right\}$, where $\mathcal{F}_{j} \ni \sum_{i \leq j, i \in \mathcal{A}} B_{i}$, and the variables $\left\{B_{i}: i \leq j, i \in \mathcal{A}\right\}$ are exchangeable with respect to $\mathcal{F}_{j}$. Then

$$
\mathbb{E}\left[\frac{1+|\{i \leq J: i \in \mathcal{A}\}|}{1+\sum_{i \leq J, i \in \mathcal{A}} B_{i}}\right] \leq \rho^{-1}
$$

Proof OF . Let $\mathcal{A}=\left\{i_{1}, \ldots, i_{m}\right\}$ where $1 \leq i_{1}<\cdots<i_{m} \leq n$. Then by considering the i.i.d. sequence

$$
B_{i_{1}}, \ldots, B_{i_{m}}
$$

in place of $B_{1}, \ldots, B_{n}$, we see that this result is equivalent to Lemma 2. 
Proof of Lemma 1. We may assume $\rho<1$ to avoid the trivial case. We now create a different construction for the $B_{i}$ 's. First, generate a random set $\mathcal{A} \subseteq[n]$ where for each $i$, independently,

$$
\mathbb{P}\{i \in \mathcal{A}\}=\frac{1-\rho_{i}}{1-\rho} .
$$

Next, define variables $Q_{1}, \ldots, Q_{n} \stackrel{\text { iid }}{\sim} \operatorname{Bernoulli}(\rho)$, which are generated independently of the random set $\mathcal{A}$. Finally, define

$$
B_{i}=Q_{i} \cdot \mathbb{1}_{i \in \mathcal{A}}+\mathbb{1}_{i \notin \mathcal{A}} .
$$

Then, clearly, the $B_{i}$ 's are mutually independent with $\mathbb{P}\left\{B_{i}=1\right\}=\rho_{i}$, as required by the lemma. Next, since $B_{i}=Q_{i} \cdot \mathbb{1}_{i \in \mathcal{A}}+\mathbb{1}_{i \notin \mathcal{A}}$ for all $i$, we have

$\frac{1+J}{1+B_{1}+\cdots+B_{J}}=\frac{1+|\{i \leq J: i \in \mathcal{A}\}|+|\{i \leq J: i \notin \mathcal{A}\}|}{1+\sum_{i \leq J, i \in \mathcal{A}} Q_{i}+|\{i \leq J: i \notin \mathcal{A}\}|} \leq \frac{1+|\{i \leq J: i \in \mathcal{A}\}|}{1+\sum_{i \leq J, i \in \mathcal{A}} Q_{i}}$,

where the last step uses the identity $\frac{a+c}{b+c} \leq \frac{a}{b}$ whenever $0<b \leq a$ and $c \geq 0$. Therefore, it will be sufficient to bound the right-hand side. We will use to prove that

$$
\mathbb{E}\left[\frac{1+|\{i \leq J: i \in \mathcal{A}\}|}{1+\sum_{i \leq J, i \in \mathcal{A}} Q_{i}} \mid \mathcal{A}\right] \leq \rho^{-1},
$$

which will be sufficient to prove the lemma via the tower law of expectations.

To prove (4), first let $\widetilde{Q}_{i}=Q_{i} \cdot \mathbb{1}_{i \in \mathcal{A}}$, and define a filtration $\left\{\mathcal{F}_{j}^{\prime}\right\}$ where $\mathcal{F}_{j}^{\prime}$ is the $\sigma$-algebra generated as

$$
\mathcal{F}_{j}^{\prime}=\sigma\left(\left\{\widetilde{Q}_{1}+\cdots+\widetilde{Q}_{j}, \widetilde{Q}_{j+1}, \ldots, \widetilde{Q}_{n}, \mathcal{A}\right\}\right) .
$$

Next, for any $j$, by (3) we see that

$$
B_{1}+\cdots+B_{j}, B_{j+1}, \ldots, B_{n} \in \mathcal{F}_{j}^{\prime} \quad \Rightarrow \quad \mathcal{F}_{j}^{\prime} \supseteq \mathcal{F}_{j},
$$

and so $T$ is a stopping time (in reverse time) with respect to $\left\{\mathcal{F}_{j}^{\prime}\right\}$ also. Next, the variables $\left\{Q_{i}: i \leq j, i \in \mathcal{A}\right\}$ are trivially exchangeable with respect to $\mathcal{F}_{j}^{\prime}$ (since the $Q_{i}$ 's are i.i.d., and independent from $\mathcal{A}$ ). Finally, since the $Q_{i}$ 's are independent from $\mathcal{A}$, the desired bound (4) follows directly from after conditioning on $\mathcal{A}$.

2. Detailed results for GWAS experiment. In this section we present the full results obtained in the GWAS experiment (see Section 6.2 in the main paper for details). Table 1 gives the results for the LDL phenotype, and Table 2 gives the results for the HDL phenotype.

imsart-aos ver. 2014/10/16 file: supplement.tex date: September 7, 2018 
Selection Selection frequency frequency (by region) (by SNP)
SNP name Chromosome

rs693

rs693
rs3923037

rs754524

rs6754295

rs 1429974

\begin{tabular}{rr}
\hline 7 & 7 \\
\hline 7 & 7 \\
\hline 7 & 7
\end{tabular}

2

(n)
rs646776

2

2

$7 \quad$ rs646776 119

$7 \quad$ rs157580

rs11668477

$2 \quad$ rs 11878377

2 rs1541596

rs 10409243

\section{6}

21232195

21148274

21311541

21206183

21300770

$\begin{array}{lr}1 & \text { rs688 } \\ 2 & \text { rs174556 }\end{array}$

$\begin{array}{ll}2 & \text { rs } 174450\end{array}$

1 rs579383

$1 \quad$ rs 1535

$\begin{array}{r}\hline 4 \\ \hline 4 \\ \hline 3 \\ \hline 3 \\ \hline 3 \\ \hline 3\end{array}$

4

rs2728487

4

rs9696070

$3 \quad$ rs557435

3 rs4906908

2 rs579163

$1 \quad$ rs518181

\begin{tabular}{cc} 
& 1 \\
\hline 2 & 2 \\
\hline 2 & 2 \\
\hline 2 & 2 \\
\hline 2 & 2 \\
\hline 2 & 1
\end{tabular}

rs 11216267

$\begin{array}{ll}1 & \text { rs } 11216267 \\ 2 & \text { rs } 2802955\end{array}$

2 rs1095354

$\begin{array}{lr}2 & \text { rs10953541 } \\ 2 & \text { rs905502 }\end{array}$

$2 \quad$ rs1897318

1 rs 1006236

2

$1 \quad$ rs6896136

1 rs 12427378

$1 \quad$ rs2139930

\begin{tabular}{lll}
\hline 1 & 1 & rs7574918 \\
\hline 1 & 1 & rs6749903 \\
\hline 1 & 1 & rs3733262 \\
\hline
\end{tabular}

\begin{tabular}{llcccc}
\hline 1 & 1 & rs3733262 & 4 & 129439663 & \\
\hline 1 & 1 & rs981862 & 5 & 93615466 & \\
\hline 1 & 1 & rs995124 & 7 & 41764992 & \\
\hline 1 & 1 & rs2351643 & 8 & 63076996 & \\
\hline 1 & 1 & rs945559 & 10 & 89823147 & \\
\hline 1 & 1 & rs1955105 & 12 & 115344782 & \\
\hline 1 & 1 & rs2456930 & 15 & 62687339 & \\
\hline 1 & 1 & rs4433842 & 17 & 64322683 & rs1801689 \\
\hline 1 & 1 & rs4799847 & 18 & 33686203 & \\
\end{tabular}

\section{TABLE 1}

Results of GWAS experiment for the LDL phenotype (see Section 6.2 in the main paper for details and interpretation of the results). Physical reference positions for SNPs were drawn from Human

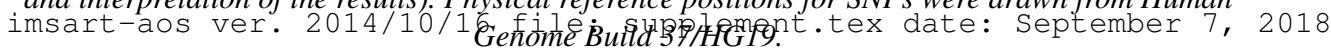




\section{SUPPLEMENTARY MATERIAL: A KNOCKOFF FILTER FOR HIGH-DIMENSIONAL SELECTIVE INFERENCE}

\begin{tabular}{|c|c|c|c|c|c|c|}
\hline $\begin{array}{l}\text { Selection } \\
\text { frequency } \\
\text { (by region) }\end{array}$ & $\begin{array}{l}\text { Selection } \\
\text { frequency } \\
\text { (by SNP) }\end{array}$ & SNP name & Chromosome & $\begin{array}{c}\text { Base pair } \\
\text { position }\end{array}$ & $\begin{array}{l}\text { Nearby SNPs } \\
\quad \text { found in } \\
\text { Willer et al. [3] }\end{array}$ & $\begin{array}{l}\text { Nearby SNPs } \\
\text { found in } \\
\text { Sabatti et al. [2] }\end{array}$ \\
\hline \multirow[t]{2}{*}{9} & 9 & rs3764261 & 16 & 56993324 & rs3764261 & rs3764261 \\
\hline & 9 & rs7499892 & & 57006590 & & \\
\hline 8 & 8 & rs1532085 & 15 & 58683366 & rs1532085 & rs 1532085 \\
\hline \multirow[t]{2}{*}{4} & 2 & rs 2575875 & 9 & 107662494 & rs 1883025 & \\
\hline & 2 & rs 2740486 & & 107666513 & & \\
\hline \multirow[t]{2}{*}{4} & 2 & rs255049 & 16 & 68013471 & rs16942887 & rs 255049 \\
\hline & 2 & rs 255052 & & 68024995 & & \\
\hline \multirow[t]{2}{*}{3} & 3 & rs7120118 & 11 & 47286290 & rs3136441 & rs2167079 \\
\hline & & & & & & rs7120118 \\
\hline 1 & 1 & rs 12139970 & 1 & 230406460 & rs4846914 & \\
\hline 1 & 1 & rs6728178 & 2 & 21193946 & & \\
\hline 1 & 1 & rs173738 & 5 & 16725880 & & \\
\hline 1 & 1 & rs2817056 & 6 & 35734051 & & \\
\hline 1 & 1 & rs2375016 & 12 & 109227914 & rs7134594 & \\
\hline
\end{tabular}

Results of GWAS experiment for the HDL phenotype (see Section 6.2 in the main paper for details and interpretation of the results). Physical reference positions for SNPS were drawn from Human Genome Build 37/HG19.

\section{References.}

[1] Rina Foygel Barber and Emmanuel J. Candès. Controlling the false discovery rate via knockoffs. Ann. Statist., 43(5):2055-2085, 2015.

[2] Chiara Sabatti, Susan K Service, Anna-Liisa Hartikainen, Anneli Pouta, Samuli Ripatti, Jae Brodsky, Chris G Jones, Noah A Zaitlen, Teppo Varilo, Marika Kaakinen, Ulla Sovio, Aimo Ruokonen, Jaana Laitinen, Eveliina Jakkula, Lachlan Coin, Clive Hoggart, Andrew Collins, Hannu Turunen, Stacey Gabriel, Paul Elliot, Mark I McCarthy, Mark J Daly, Marjo-Riitta Järvelin, Nelson B Freimer, and Leena Peltonen. Genome-wide association analysis of metabolic traits in a birth cohort from a founder population. Nature genetics, 41(1):35-46, 2009.

[3] Cristen J. Willer, Ellen M. Schmidt, Sebanti Sengupta, et al. Discovery and refinement of loci associated with lipid levels. Nature genetics, 45(11):1274-1283, 2013.

E-MAIL: rina@uchicago.eduDePARTMENT of STATISTICS, UNIVERSITY of CHICAGO E-MAIL: candes@ stanford.eduDEPARTMENTS OF STATISTI 